\title{
Are LINERs Starbursts or Mini-quasars? A comparative Study of their Ultraviolet Spectra
}

\author{
Anuradha Koratkar \\ Space Telescope Science Institute
}

\begin{abstract}
.
Low Ionization Nuclear Emission Line Regions (LINERs) are found in $\sim 30 \%$ of all bright galaxies. The nuclear luminosities in these objects are such that they can be produced by a number of mechanisms and there have been heated debates on the nature of ionizing sources in LINERs. The variety of ionizing mechanisms suggested are low luminosity AGNs, starbursts, shocks, or any combination of these. We have studied Hubble Space Telescope (HST) ultraviolet (UV) spectra of seven LINERs having compact nuclear UV sources.

The picture emerging from this comparison is that the compact source observed in these LINER galaxies, at least in some cases, is a nuclear star cluster rather than a low-luminosity active galactic nucleus (AGN). In these cases, the UV luminosity is driven by tens of thousands of O-type stars, depending on the assumed extinction for these objects. The O-stars could be the high-mass end of a bound stellar population, similar to those seen in super star clusters. Our data do not exclude the possibility that a similar stellar continuum source could dominate in all the LINERs. Alternatively, there may be two types of UV-bright LINERs: those where the UV continuum is produced by a starburst, and those where it is nonstellar.

The "clearly-stellar", weak [O I] emitters, LINERs have relatively weak X-ray emission, and their stellar populations probably provide enough ionizing photons to explain the observed optical emission-line flux. The other LINERs, strong [O I] emitters, have severe ionizing photon deficits, for reasonable extrapolations of their UV spectra beyond the Lyman limit, but have an X-ray/UV power ratio that is higher by two orders of magnitudes than that of the "clearly-stellar" LINERs. A component which emits primarily in the extreme-UV may be the main photoionizing agent in these objects.

Recent results show that nuclear-starburst and quasar-like activity are often intermingled. Our results extend this result to the lower luminosities of the LINERs.
\end{abstract}

\section{Why study LINERs?}

Low Ionization Nuclear Emission Line Regions (LINERs) are galaxies which show low nuclear activity with narrow optical emission lines. Since the original 
survey of bright galaxies by Heckman (1980), several other surveys (see Ho 1996 for a review) have established that LINER activity is found in $\sim 30 \%$ of the nearby galaxies. Thus, the LINER phenomenon is the commonest form of nuclear activity in galaxies.

The luminosities of most LINERs are unimpressive compared to "classical" AGNs. The nuclear luminosities in these objects are such that they can be produced by a number of mechanisms and there have been heated debates on the nature of ionizing source in LINERs. Several other ionizing mechanisms such as low luminosity AGNs (Ferland \& Netzer 1983), hot high-metallicity O-type stars (Filippenko \& Terlevich 1992; Shields 1992), radiative shocks in accretion flows or winds (Dopita \& Sutherland 1995), or UV-bright post-AGB stars (Binette et al. 1994) have been invoked as the energy sources of LINERs.

If LINERs represent the low-luminosity end of the AGN phenomenon, then they are the nearest and most common examples, and their proximity can be used to get a fundamental understanding of the AGN phenomenon. Their study can also be used to understand the connection between AGNs and starbursts.

What is the nature of the energy source powering LINERs? Are LINERs the low luminosity end of AGNs? These are some of the fundamental questions still unanswered. To properly address these questions, the various proposed energy sources have to be distinguished observationally, and their energy contribution quantified. Studies involving only optical emission line ratios are not capable of differentiating among the several proposed ionizing mechanisms. Ideally multi wavelength observations are preferred, but the UV is a good starting place as it provides a number of discriminants. The $\mathrm{x}$-ray and IR investigations are just starting to achieve the high spatial and spectral resolution demanded by these investigations.

\section{UV spectroscopy of LINERs}

When a search for UV point sources in LINERS was conducted, only $\sim 22 \%$ of the LINERs showed a compact UV-bright source with HST (Maoz et al. 1996a; Barth et al. $1996 ; 1998)$. Since internal extinction effects play a crucial role in the detection of UV-bright sources, this fraction is a lower limit of the total fraction of LINERs with a central UV compact source. The compactness of the sources suggested that they could either be nonstellar in nature, or compact star clusters. The UV luminosity in these compact sources was consistent with the object being a low luminosity AGN, star cluster or shock. UV spectroscopy can be used to distinguish the ionizing mechanism, since all these ionizing mechanisms have UV spectral diagnostics. I present UV (1150-3200 $\AA)$ spectra for seven LINERs and compare their properties.

Figure 1 shows the $1200-1590 \AA$ spectra for the seven LINERs. We immediately see that only two of these LINERs have broad emission lines similar to those seen in "classical" AGN. Except for the two LINERs with broad emission lines (M81 and NGC 4579), the LINERs have weak or no detectable UV emission lines.

The spectra of NGC 404, NGC 4569, and NGC 5055 show clear absorptionline signatures of massive stars, indicating a stellar origin for the UV continuum. The UV luminosity, in these object, is driven by tens to thousands of O-type 


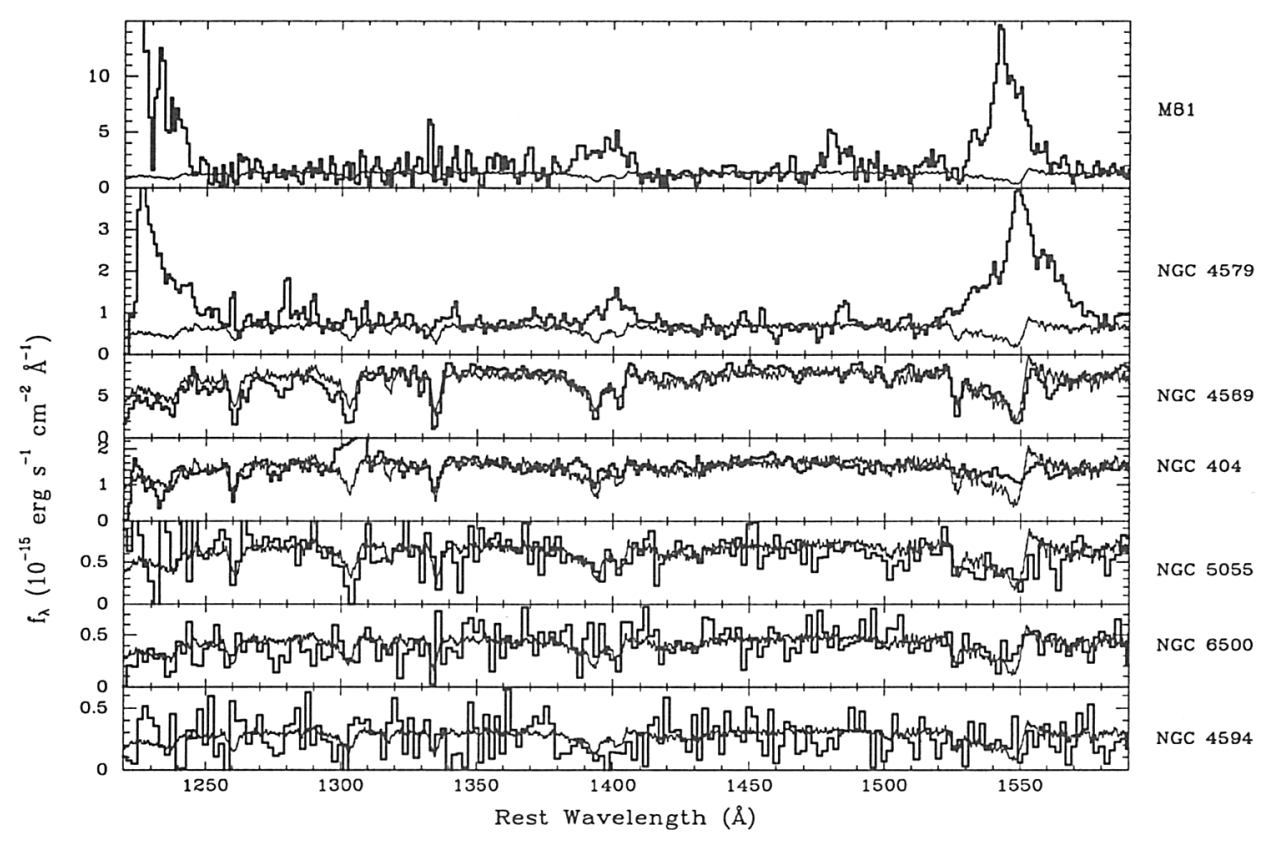

Figure 1. The UV spectra of seven LINERs (bold line), ordered with the two broad-lined objects on the top, and then in decreasing $f_{\lambda}$. The spectrum of the starburst NGC 1741-B is overlayed in each case. The starburst spectrum is normalized to be flat in $f_{\lambda}$ and scaled to match the LINER continuum level. The compact central UV continuum source that is observed in these galaxies is a nuclear star cluster rather than a low-luminosity AGN, at least in some cases. The same stellar signatures may be present but undetectable in M81 and NGC 4579 , due to superposed strong, broad emission lines.

stars, depending on the object and the extinction assumed. The O-stars could be the high-mass end of a bound stellar population, similar to those seen in super star clusters (e.g., Maoz et al. 1996b) . Spectral signatures of massive stars are probably also present in NGC 6500 . The same stellar signatures may be present but undetectable in NGC 4594, due to the low signal-to-noise ratio of the spectrum, and in M81 and NGC 4579, due to superposed strong, broad emission lines. Thus the UV continuum in all these objects could have a stellar origin. Circumnuclear starbursts within $300 \mathrm{pc}$. of the galaxy nucleus are often seen and these can contribute significantly in the UV. But is the observed stellar continuum sufficient to power the LINERs?

A comparison of the $\mathrm{H} \alpha$ line flux to the continuum flux at $1300 \AA$ indicates that the emission line strengths are not correlated to the observed UV continuum, indicating that the emission lines are not driven by the observed UV continuum. To determine if the stellar continuum was sufficient to power the LINERs we computed the ionizing photons due to young star clusters. We found that in the three LINERs whose UV emission is clearly dominated by 
stars (NGC 404, NGC 4569, and NGC 5055), ionization by the stellar population can provide the required power, but only if very massive stars are still present. Incidentally these objects are also weak [O I] emitters. In the other four LINERs (strong [O I] emitters) there was a ionizing photon deficit, indicating an additional energy source beyond that implied by the observable UV.

To search for an additional source of photoionizing photons we compared the UV flux with the $2-10 \mathrm{keV}$ X-ray flux. We found that the strong [O I] emitters have X-ray power comparable to the UV power. The weak [O I] emitters have X-ray power which is at least an order of magnitude lower than the UV. Thus the strong [O I] emitters have an ionizing source which dominates in the $\mathrm{X}$-ray and Extreme-UV. Thus, a non-stellar source may be significant or even dominate at other wavelengths. Although the three "clearly-stellar" LINERs do not obviously require the existence of such an additional ionizing source, our data do not exclude the existence of such a source.

Acknowledgments. This work was supported by HST GO grant GO-6112 provided by the Space Telescope Science Institute, which is operated by the Association of Universities for Research in Astronomy Inc., under NASA contract NAS 5-26555.

\section{References}

Barth, A.J., Ho, L.C., Filippenko, A.V., \& Sargent, W.L.W. 1996, in "The Physics of LINERs in View of Recent Observations", eds. M. Eracleous et al. (San Francisco: ASP), p. 153

Barth, A.J., Ho, L.C., Filippenko, A.V., \& Sargent, W.L.W. 1998, ApJ, 496, 133

Binette, L., Magris, G., Stasinska, G., \& Bruzual, G. 1994, A\&A, 292, 13

Dopita, M., \& Sutherland, R. 1995, Ap.J., , 455, 468

Ferland, G. J., \& Netzer, H. 1983, ApJ, 264, 105

Filippenko, A. V., \& Terlevich, R. 1992, ApJ, 397, L79

Heckman, T.M. 1980, A\&A, 87, 152

Ho, L.C., Filippenko, A.V., \& Sargent, W.L.W. 1996, ApJ, 462, 183

Maoz, D., Filippenko, A.V., Ho, L.C., Macchetto, F.D., Rix, H.-W., \& Schneider, D.P. 1996a, ApJS, 107, 215

Maoz, D., Barth, A.J., Sternberg, A., Filippenko, A.V., Ho, L.C., Macchetto, F.D., Rix, H.-W., \& Schneider, D.P. 1996b, AJ, 111, 2248 